\title{
Assistência nutricional para os cuidados paliativos de pacientes oncológicos: uma revisão integrativa
}

\author{
Nutritional assistance to palliative care of oncological patients: an integrative review
}

Ennya Cristina Pereira dos Santos Duarte ${ }^{a}$

Orcid: https://orcid.org/0000-0001-6096-5259

Rosana Rodrigues de Sousa ${ }^{b}$

Orcid: https://orcid.org/0000-0002-7426-2471

\author{
Maria Clara Feijó-Figueiredo ${ }^{c}$ \\ Orcid: https://orcid.org/0000-0001-5982-6868
}

Joilane Alves Pereira-Freire ${ }^{d}$

Orcid: https://orcid.org/0000-0003-0596-934X

\begin{abstract}
Resumo
Introdução: $\mathrm{O}$ acompanhamento nutricional é fundamental na aplicabilidade dos cuidados paliativos no câncer avançado. Tais cuidados visam minimizar os efeitos adversos dos tratamentos e propiciar melhoria à qualidade de vida de pacientes e familiares, aliviando $o$ sofrimento subjetivo. Objetivo: Avaliar a influência da assistência nutricional para os cuidados paliativos de pacientes oncológicos. Metodologia: Trata-se de uma revisão integrativa da literatura. Para a busca dos artigos foram utilizadas as combinações dos termos "câncer", "cuidados paliativos", "nutrição" ou "terapia nutricional" (cancer and palliative care and nutrition or nutrition therapy), nas bases de dados Pubmed, Scielo e Lilacs, no período de 2009 a 2019. Resultados: Após triagem, foram incluídos nove estudos que investigaram o estado nutricional, especialmente no âmbito da síndrome de caquexia-anorexia, os significados do alimento e da nutrição para o paciente, o uso de terapia nutricional enteral ou parenteral, as contribuições da suplementação nutricional e condução do acompanhamento nutricional pelos profissionais. A nutrição e a alimentação em todos os seus aspectos mostraram-se relevantes no tratamento por contribuir para a melhora da qualidade de vida, quer seja por meio de estratégias nutricionais como a terapia nutricional por via enteral e/ou parenteral, na impossibilidade da via oral, ou na suplementação, o que possibilita resposta positiva no estado nutricional, retarda a progressão da caquexia e reduz sintomas gastrointestinais. Conclusão: $\mathrm{O}$ acompanhamento nutricional deve ser adequado, respeitando as necessidades biológicas e as relações socioantropológicas do paciente com a alimentação, além de seus anseios, sem desconsiderar as condições clínicas e todos os aspectos éticos envolvidos no processo.

Palavras-chave: câncer; cuidados paliativos; nutrição; terapia nutricional.
\end{abstract}

\begin{abstract}
Introduction: Nutritional monitoring is essential in the application of palliative care in advanced cancer. Such care aims to reduce the adverse effects of treatments and improve the quality of life of patients and families, relieving or suffering subjective suffering. Objective: To evaluate the influence of nutritional assistance for palliative care of cancer patients
\end{abstract}

\footnotetext{
a Graduação em Nutrição, Mestre em Alimentos e Nutrição e discente do Curso de Especialização em Oncologia

Experimental e Clínica do Programa de Pós-graduação em Ciências Farmacêuticas, do Centro de Ciências da Saúde, Teresina - Piauí. Universidade Federal do Piauí, Brasil. E-mail: ennyacristina@hotmail.com

${ }^{\text {b }}$ Graduação em Nutrição, Mestre em Alimentos e Nutrição, Departamento de Nutrição, Centro de Ciências da Saúde,

Teresina - Piauí. Universidade Federal do Piauí, Brasil. E-mail: rosanasousa@ufpi.edu.br

${ }^{c}$ Discente do Curso de Nutrição, Departamento de Nutrição, Campus Senador Helvídio Nunes de Barros, Picos - Piauí.

Universidade Federal do Piauí. Brasil. E-mail: clara.37.m@gmail.com

${ }^{d}$ Graduação em Nutrição, Doutora em Biotecnologia em Saúde, Professora Adjunta da Universidade Federal do Piauí

(UFPI), Curso de Nutrição, Campus Senador Helvídio Nunes de Barros, Picos - Piauí. Universidade Federal do Piauí, Brasil.

E-mail: joilane@ufpi.edu.br
} 
Methodology: This is an integrative literature review. To search for the articles, combinations of the terms "cancer", "palliative care", "nutrition" or "nutrition therapy" were used in the Pubmed, Scielo and Lilacs databases, considering the period from 2009 to 2019. Results: After screening, nine studies were investigated that investigated the nutritional status, especially in the context of cachexia-anorexia syndrome, the meanings of food and nutrition for the patient, the use of enteral or parenteral nutritional therapy, the contributions of nutritional supplementation and conducting nutritional monitoring by professionals. Nutrition and food in all its aspects proved to be relevant in the treatment because it contributes to the improvement of the quality of life, whether through nutritional strategies such as enteral and / or parenteral nutritional therapy, when the oral route is impossible, or in supplementation, which allows a positive response in nutritional status, slows the progression of cachexia and reduces gastrointestinal symptoms. Conclusion: Nutritional monitoring must be adequate, respecting the patient's biological needs and socio-anthropological relationships with food, in addition their desires without disregarding clinical conditions and all ethical aspects involved in the process.

Keywords: cancer; palliative care; nutrition; nutritional therapy.

\section{Introdução}

Dentre as doenças crônicas com grande incidência no Brasil e no mundo, o câncer destaca-se como uma das que apresentam a maior taxa de mortalidade, levando à morte, aproximadamente, 8,8 milhões de pessoas no mundo no ano de 2015. De acordo com a OMS, o câncer é a segunda principal causa de morte no mundo, o qual é responsável por 9,6 milhões de mortes em 2018 e a nível global, uma em cada seis mortes são relacionadas à doença ${ }^{1}$.

Para os casos de estado avançado e na impossibilidade de cura, a OMS descreve um tipo de tratamento, denominado Tratamento paliativo, o qual visa minimizar os efeitos adversos dos tratamentos médicos e que gerem melhoria à qualidade de vida dos pacientes e de seus familiares, com o objetivo de alívio do sofrimento e humanização dos cuidados ao paciente ${ }^{2}$.

Os cuidados paliativos consistem em uma forma de cuidar altamente estruturada, que visam fornecer atendimento humanizado aos pacientes e também aos familiares diante de situações de enfermidades que ponham em risco a vida. O programa de tratamentos paliativos exige do profissional de saúde uma preparação adequada, para que ele possa agir diante do conjunto de problemas que estão sendo enfrentados pela família e pelo paciente, como o sofrimento mental, físico, social e psicológico ${ }^{3}$.

Os pacientes em estado de doenças avançadas, como no caso do câncer, sofrem de muitos sintomas decorrentes não só da enfermidade, mas também dos grandes efeitos colaterais causados pelo tratamento de quimioterapia e radioterapia. Dentre esses sintomas, destacam-se as alterações fisiológicas que impedem o paciente de se alimentar normalmente, as quais podem favorecer quadros de anorexia, decorrentes, sobretudo, da síndrome de caquexia, a qual é muito frequente em pacientes com câncer terminal. Desse modo, a nutrição inadequada do paciente oncológico contribui para a piora no prognóstico da doença e reduz a sua qualidade de vida do mesmo, tornando o tratamento muito mais doloroso e demorado ${ }^{4}$.

O acompanhamento nutricional é fundamental na aplicabilidade dos cuidados paliativos aos pacientes oncológicos, como o uso da alimentação artificial, que auxilia na melhora do balanço energético e desacelera o processo de caquexia, atuando na manutenção do peso e redução do processo catabólico. Logo, o papel do nutricionista no tratamento paliativo transforma a função meramente fisiológica de nutrir e atua nos aspectos social e psicológico, propiciando a melhora do estado de isolamento social e o enfrentamento do estágio terminal do câncer de maneira menos dolorosa ${ }^{2}$.

É essencial que o nutricionista esteja atento à importância da assistência nutricional no tratamento paliativo e da sua inserção nas equipes voltadas aos cuidados paliativos, para que em conjunto com outros profissionais da saúde possibilite maior qualidade de vida durante o processo patológico. Assim, esse 
estudo teve por objetivo avaliar a influência da assistência nutricional para os cuidados paliativos de pacientes oncológicos.

Este estudo trata de uma revisão integrativa. Segundo Roman e Friedlander ${ }^{5}$, a revisão integrativa de pesquisa possibilita separar o achado científico de opiniões e ideias e, além de descrever o conhecimento no seu estado atual, promove o impacto da pesquisa sobre a prática profissional. Permite ainda, fazer generalizações sobre assuntos específicos estudados por vários pesquisadores, em vários lugares e momentos distintos, mantendo os interessados atualizados e facilitando as modificações da prática cotidiana como consequência da pesquisa.

Esse tipo de revisão permite a inclusão de estudos experimentais e nãoexperimentais, além de combinar dados da literatura teórica e empírica, e um vasto leque de propósitos: definição de conceitos, revisão de teorias e evidências, e análise de problemas metodológicos de um tópico particular ${ }^{6}$.

A pergunta que motiva esta revisão é: "Qual a influência da assistência nutricional no processo de cuidados paliativos do paciente oncológico?". A partir desse questionamento foram definidos, segundo o Desc Saúde, os seguintes descritores: câncer, cuidados paliativos, nutrição, terapia nutricional. Para a busca dos artigos foram utilizadas as combinações desses termos nas bases de dados Pubmed, Scielo e Lilacs, considerando-se o período de 2009 a 2019.

Após a realização da busca, os artigos passaram por triagem após leitura do título e/ou resumo, considerando-se os seguintes critérios para que fosse selecionado para a leitura integral: publicação em inglês, português ou espanhol, texto disponíveis na íntegra, não se tratar de revisões bibliográficas, abordar aspectos nutricionais (suplementação, estado nutricional, terapia nutricional, dentre outros) relacionados ao processo de cuidados paliativos de pacientes oncológicos. Após a leitura do título e resumo dos textos selecionados, foram também excluídos os artigos duplicados nas bases de dados pesquisadas.

Para a leitura do texto integral foram selecionados dois (2) artigos no Scielo, seis (6) artigos no Lilacs e oito (8) artigos no Pubmed. Após a leitura aprofundada foram incluídos apenas nove (9) estudos neste estudo (Figura 01).

Figura 01- Fluxograma da busca e seleção dos artigos para inclusão no estudo.

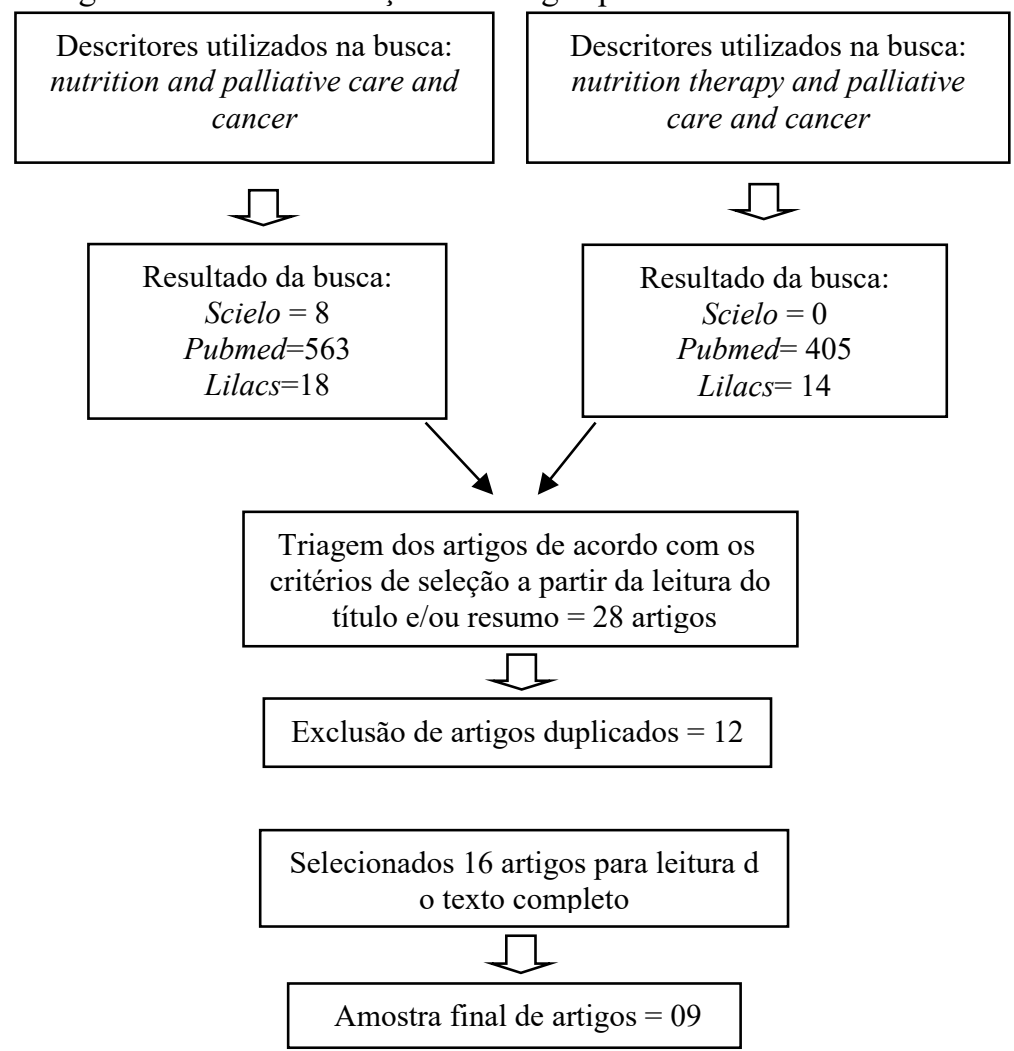




\section{Desenvolvimento}

A amostra final de artigos resultou em 3 artigos em português, 1 em espanhol e 5 em inglês, publicados entre 2010 e 2018, frutos de pesquisas que relacionaram aspectos nutricionais de forma direta ou indireta com o tratamento paliativo do câncer, em diversos países como Brasil, Suécia, Índia, Alemanha e México.

Esses estudos investigaram o estado nutricional, especialmente no âmbito da síndrome de caquexia-anorexia, além da sarcopenia, os significados do alimento e da nutrição para o paciente, o uso de terapia nutricional enteral ou parenteral, as contribuições da suplementação nutricional, além de como é realizado o acompanhamento nutricional desses pacientes. O Quadro 01 (Apêndice) lapresenta os artigos incluídos neste estudo, bem como seus objetivos e respectivos resultados e/ou conclusões.

Camargo et $\mathrm{al}^{7} \mathrm{e}$ Queiroz et $\mathrm{al}^{8}$ estudaram a respeito do estado nutricional de pacientes oncológicos adultos e idosos em cuidados paliativos e sua relação com enfoque em fatores que têm impacto na qualidade de vida dos mesmos como a sarcopenia e a síndrome da anorexiacaquexia.

Em relação à sarcopenia, Queiroz et $\mathrm{al}^{8}$ concluíram que essa condição está associada ao pior estado nutricional e potencialização do comprometimento funcional. Assim, os pacientes que possuem diminuição da massa muscular, devido à sarcopenia, apresentam menores escores de qualidade de vida, comparados àqueles sem déficit de massa muscular ${ }^{9}$.

Dessa forma, é importante ressaltar que uma avaliação multidimensional detalhada é a base da tomada de decisão em pacientes com câncer avançado, que sofrem de anorexia, caquexia e outros sintomas relacionados, os quais afetam diretamente as esferas física, psicossocial e existencial ${ }^{8}$.

A síndrome da anorexia-caquexia é caracterizada por perda de apetite e peso, distúrbios metabólicos e processos inflamatórios. Alterações metabólicas secundárias a essa síndrome impedem que pacientes caquéticos ganhem peso, recuperem massa corporal e diminuam a sobrevida, além de ser clinicamente importante, pois influencia a resposta aos tratamentos de quimioterapia e radioterapia ${ }^{10}$.

$$
\text { Kapoor et } \mathrm{al}^{11} \text { consideraram o }
$$

papel da nutrição essencial para o tratamento da caquexia. Os resultados de uma intervenção realizada, em que a associação de atividade física leve, orientação nutricional e suplementação com uma mistura de farinhas de grama de bengala torrada, cevada torrada, soja torrada, pó de linhaça e pó de Amaranthus spinosus seco, apontaram que essa suplementação nutricional na terapia de cuidados paliativos pode melhorar a qualidade de vida e estabilizar o peso corporal em pacientes com caquexia por câncer.

De acordo com Camargo et $\mathrm{al}^{7}$, a alta prevalência de desnutrição entre pacientes oncológicos é devida à síndrome da anorexia-caquexia, que está associada a sintomas gastrointestinais como: náuseas, xerostomia, saciedade precoce e perda de apetite, além de disgeusia, disfagia, diarreia ou constipação que intensificam o estado nutricional deficiente.

Contudo, algumas condutas nutricionais podem ser adotadas para atenuar sintomas gastrintestinais e ajudar no controle de náuseas e vômitos como: fracionar as refeições; evitar odores e temperos fortes, temperaturas extremas, além de orientar a não ingerir líquidos durante as refeições e não consumir alimentos açucarados.

A constipação é outra manifestação muito recorrente, principalmente pelo uso de opioides para controle da dor. Nesse sentido, a orientação é o aumento do consumo de líquidos associado a diferentes tipos de fibras, a exemplo de hortaliças, cereais integrais e frutas, alimentos que otimizam a motilidade 
intestinal e $\mathrm{o}$ funcionamento do trato gastrointestinal $^{12,13}$.

Ressalta-se ainda a relevância do cuidado nutricional precoce em pacientes com câncer paliativo para avanço na qualidade de vida ${ }^{14}$. As causas reversíveis de anorexia e caquexia devem ser identificadas e tratadas sempre que possível $^{7}$. Desse modo, a uniformização dos critérios diagnósticos e a elaboração de protocolos de assistência nutricional específicos para essa população, podem refletir em melhora da qualidade de vida, sendo esse o principal objetivo dos cuidados paliativos $^{8}$.

Segundo Silva et $\mathrm{al}^{15}$, a
interdisciplinar em pacientes oncológicos em cuidados paliativos pretende contribuir com o controle da dor e os demais sintomas decorrentes da neoplasia. O paciente deve ser visto de maneira holística, para que o mesmo tenha condições de possuir o mínimo de autonomia. $\mathrm{O}$ atendimento médico e nutricional de forma integrada permite escolhas assertivas quanto à conduta medicamentosa e dietoterápica, com consequente redução dos efeitos colaterais possíveis dos medicamentos. Portanto, a intervenção precoce nutricional permite monitorar adequadamente o tipo de dieta ofertada e o volume de alimentos, e assim melhorar o controle dos sintomas.

O profissional de nutrição deve planejar sua prescrição clínica visando ofertar as quantidades adequadas de nutrientes, respeitando necessidades calóricas, desejos e preferências nutricionais dos pacientes, bem como a diminuição da ingestão de alimentos na fase final da vida ${ }^{16}$.

Considerando os estudos de Vetori, Santos e Peria ${ }^{16}$ e de Silva et $\mathrm{al}^{15}$ é possível inferir que o nutricionista é extremante necessário para o processo de cuidados paliativos de pacientes oncológicos, pois a abordagem nutricional escolhida impactará diretamente na qualidade de vida do paciente. Então, a inserção do nutricionista na equipe de cuidados paliativos é fundamental, principalmente por exercer funções consideráveis no acompanhamento dos pacientes internados ${ }^{17,2}$.

Além disso, o alimento não possui caráter apenas biológico, mas também social e cultural, repleto de significados. Em estudo realizado por Costa e Soares ${ }^{18}$, desenvolvido no Brasil e em Portugal, os significados do alimento e da alimentação para os pacientes em tratamento oncológico que recebem cuidados paliativos refletem a importância que é dada ao ato de se alimentar, destacado tanto por pacientes quanto por familiares como algo vital, somado ao fato que para esses indivíduos não existe diferenciação entre os termos alimento e nutrição. Consequentemente, a alimentação nos cuidados paliativos oncológicos possui influência de muitas variáveis e está associada à saúde e à qualidade de vida.

A American Dietetic Association ${ }^{19}$ recomenda que a alimentação via oral seja priorizada, desde que o sistema gastrointestinal esteja íntegro, o paciente apresente desejo e possua condições clínicas para se alimentar por essa via. Porém, em algumas situações esses critérios não são atendidos e adotam-se outras vias para a alimentação como a via enteral ou a via parenteral.

No que concerne ao uso de terapia nutricional enteral ou parenteral, esta revisão elencou alguns estudos ${ }^{20,21,22}$ que investigaram algum tipo de relação dessas vias de alimentação com possíveis benefícios para o processo de cuidado paliativo em pacientes oncológicos.

Em relação à terapia nutricional enteral, Grilo, Santos e Fonseca ${ }^{22}$ verificaram que o suporte nutricional via enteral, por meio de gastrostomia percutânea endoscópica, consiste numa opção viável para pacientes com câncer avançado do esôfago e sem expectativa de retomar a ingestão oral.

No estudo de Pelzer et $\mathrm{al}^{20}$ investigou-se o impacto de nutrição parenteral sobre o estado nutricional em 
pacientes com câncer avançado de pâncreas, usando parâmetros da Bioimpedância Elétrica (BIA) como o ângulo de fase, o índice de ECM / BCM e IMC e obteve-se resultados positivos de que essa abordagem tem impacto sobre o estado nutricional, com melhora dos parâmetros avaliados. Porém, no estudo de Orrevall et $\mathrm{al}^{21}$, que investigou a relação dos benefícios do suporte nutricional parenteral em pacientes oncológicos em cuidados paliativos, o entendimento foi limitado, indicando que os resultados devem ser interpretados com cautela.

\section{Conclusão}

Os cuidados paliativos para o paciente oncológico visam proporcionar alívio da dor e conforto, benefícios que devem ser estendidos também à família do paciente. A nutrição e alimentação, em todos os seus aspectos, mostram-se como um fator relevante na melhora da qualidade de vida, por meio de estratégias nutricionais como suplementação, aporte nutricional via oral, enteral e/ou parenteral, favorecendo positivamente o estado nutricional.

Ademais, destaca-se que uma adequada assistência nutricional é indispensável para os cuidados paliativos oncológicos, pois retarda a progressão da caquexia, e permite controle mais efetivo dos sintomas gastrointestinais.

A relação do homem com 0 alimento tem início a partir do seu nascimento e não se extingue com a proximidade do final de sua vida, devendose respeitar as necessidades biológicas e as relações socioantropológicas do paciente com a alimentação e seus anseios, sem desconsiderar as condições clínicas e todos os aspectos éticos envolvidos no processo.

\section{Referências}

1. Magalhães ES, Oliveira AEM, Cunha NB. Atuação do Nutricionista para Melhora da Qualidade de Vida de Pacientes Oncológicos em Cuidados Paliativos. Arquivos de Ciências da Saúde. 2018; 25(3), 4-9.

2. Benarroz MDO, Faillace GBD, Barbosa LA. Bioética e nutrição em cuidados paliativos oncológicos em adultos. Cadernos de Saúde Pública. 2009; 25(9), 1875-1882.

3. Moritz, RD et al. Terminalidade e cuidados paliativos na unidade de terapia intensiva. Revista Brasileira de terapia intensiva. 2008; 20(4), 422-428.

4. Pinto, IF et al. Protocolo do Estudo de Caso Qualitativo "Assistência Alimentar e Nutricional em Cuidados Paliativos”. Rev. Bras. em Promoção da Saúde. 2018; 31(1),1-6.

5. Roman AR, Friedlander MR. Revisão integrativa de pesquisa aplicada à enfermagem. Cogitare Enferm. 1998; 3(2),109-12.

6. Whittemore R, Knafl K. The integrative review: update methodology. J Adv Nurs. 2005; 52(5), 546-53.

7. 7.Camargo DAP, Pérez SRA, García AM, Luigina De Nicola Delfin LN, Mendoza ETC, López MSS, García MKF; Avilés EV. Frecuencia de anorexia-caquexia y su asociación con síntomas gastrointestinales, en pacientes paliativos del Instituto Nacional de Cancerología, México. Nutrición Hospitalaria. 2014; 30(4), 891-895.

8. Queiroz MDSC, Wiegert EVM, Lima LC, De Oliveira LC. Associação entre sarcopenia, estado nutricional e qualidade de vida em pacientes com câncer avançado em cuidados paliativos. Revista Brasileira de Cancerologia. (2018); 64(1), 69-75.

9. Frio CC, Pretto, ADB, Gonzalez MC, Pastore, CA. The Influence of Body Composition on Cancer Patients' Quality of Life. Rev Bras Cancerologia. 2015;61(4), 351-357. 
10. Pérez CDA, Cinzeiros UVI, Verástegui AE; Allende PS. Suporte nutricional e manejo dos sintomas antes da quimioterapia, em pacientes com câncer de cólon avançado inicialmente fora do tratamento do câncer: relato de caso. Gazeta Mexicana de Oncologia. 2013a; 12(4), 286-290.

11. Kapoor N, Naufahu J, Tewfik S, Bhatnagar S, Garg R, Tewfik IA. Prospective Randomized Controlled Trial to Study the Impact of a Nutrition-Sensitive Intervention on Adult Women With Cancer Cachexia Undergoing Palliative Care in India. Integrative Cancer Therapies. 2017; 6(1), $74-84$.

12. Elliott L, Molseed LL, Mccallum PD, Grant B. The clinical guide to oncology nutrition. 2nd Ed. Chicago: American Dietetic Association; 2006.

13. Bachmann $P$ et al. Standards, options et recommandations: nutrition en situation palliative ou terminale de l'adulte porteur de cancer évolutif. Bull Cancer. 2001; 88, 985-1006.

14. Pérez CDA, Allende OS, Domínguez OG; Verástegui AE. Alimentação e hidratação em Medicina Paliativa. Gazeta Mexicana de Oncologia. 2013b; 12(4), 267-275.

15. Silva PB, Lopes M, Trindade LCT, Yamanouchi CN. Controle dos sintomas e intervenção nutricional. Fatores que interferem na qualidade de vida de pacientes oncológicos em cuidados paliativos. Revista Dor. 2010; 11(4), 282-288.

16. Vettori JC, Santos AFJ, Peria FM. Advanced cancer: nutritional impact and the importance of integrating palliative care in a public health service. Medicina (Ribeirão Preto Online). 2018; 51(3), 167-176.

17. Marín Caro MM, Laviano A, Pichard C. Nutritional intervention and quality of life in adult oncology patients. Clini Nutr. 2007; 26(3), 289-301.

18. Costa MF, Soares JC. Alimentar e Nutrir: Sentidos e Significados em Cuidados Paliativos Oncológicos. Revista Brasileira de Cancerologia. 2016; 62(3), 215-224.

19. American Dietetic Association. Position of the American Dietetic Association: issues in feeding the terminally ill adult. J Am Diet Assoc. 1992; 92(8), 996-1002.

20. Pelzer U, Arnold D, Goevercin M, Stieler J, Doerken B, Riess H, Oettle H. Parenteral nutrition support for patients with pancreatic cancer. Results of a phase II study. BMC Cancer. 2010; 10(86).

21. Orrevall Y et al. A National Observational Study of the Prevalence and Use of Enteral Tube Feeding, Parenteral Nutrition and Intravenous Glucose in Cancer Patients Enrolled in Specialized Palliative Care. Nutrients. 2013; 5(1), 267-282.

22. Grilo A, Santos CA, Fonseca JA. Gastrostomia percutânea endoscópica na paliação nutricional do câncer do esôfago proximal sem possibilidade de colocação de prótese. Arq Gastroenterol.2012; 49(3), 227-31.

\section{Como citar este artigo:}

Duarte ECPS, Sousa RR, Feijó-Figueiredo MC, Pereira-Freire JA. Assistência nutricional para os cuidados paliativos de pacientes oncológicos: uma revisão integrativa. Rev. Aten. Saúde. 2020; 18(64): 124-132. 
Quadro 01- Ano, autoria e objetivos dos estudos selecionados e seus principais apontamentos referentes à importância da nutrição para os cuidados paliativos de pacientes oncológicos.

\begin{tabular}{|c|c|c|c|}
\hline Autor/Ano & Objetivo & $\begin{array}{l}\text { Principais aspectos nutricionais } \\
\text { relacionado ao tratamento } \\
\text { oncológico paliativo }\end{array}$ & Principais resultados e/ou conclusões \\
\hline $\begin{array}{l}\text { Camargo et } \\
\text { al, } 2014\end{array}$ & $\begin{array}{l}\text { Conhecer a frequência da caquexia e sua } \\
\text { associação com sintomas gastrointestinais, em } \\
\text { pacientes com câncer em condição paliativa, do } \\
\text { Instituto Nacional do Câncer, atendidos no serviço } \\
\text { de Cuidados Paliativos. }\end{array}$ & $\begin{array}{l}\text { Estado Nutricional/ } \\
\text { caquexia. }\end{array}$ & $\begin{array}{l}\text { 61\% dos pacientes apresentaram anorexia-caquexia. } 95 \% \text { apresentaram desnutrição } \\
\text { que variou de moderada a grave. Também se constatou que existe associação } \\
\text { significativa dos sintomas gastrointestinais com a anorexia-caquexia. Esses dados } \\
\text { confirmam a relevância da atenção nutricional precoce em pacientes com câncer } \\
\text { paliativo, que apresentam anorexia-caquexia em associação com sintomas } \\
\text { gastrointestinais, para alcançar uma melhor qualidade de vida. }\end{array}$ \\
\hline $\begin{array}{c}\text { Costa e } \\
\text { Soares, } \\
2017\end{array}$ & $\begin{array}{l}\text { Compreender os sentidos e significados da } \\
\text { alimentação e nutrição nos cuidados paliativos } \\
\text { oncológicos para pacientes e cuidadores } \\
\text { analisados no Brasil e em Portugal. }\end{array}$ & $\begin{array}{l}\text { Significados do alimento } \mathrm{e} \\
\text { nutrição. }\end{array}$ & $\begin{array}{l}\text { Em resposta à pergunta sobre os sentidos e significados da alimentação e nutrição: a } \\
\text { ideia central mais frequente foi se não comer, não pode viver, estando presente no } \\
\text { discurso de } 100 \% \text { dos pacientes e } 78 \% \text { dos cuidadores analisados. A ideia central } \\
\text { apresentada pelos participantes demonstra a relação condicional para a vida, } \\
\text { ressaltando ser fundamental o seu valor cultural e social, cheia de significado } \\
\text { simbólico e grande carga emocional. }\end{array}$ \\
\hline $\begin{array}{l}\text { Grilo, } \\
\text { Santos e } \\
\text { Fonseca, } \\
2018\end{array}$ & $\begin{array}{l}\text { Avaliação retrospectiva dos doentes com disfagia } \\
\text { por câncer avançado do esôfago em que a } \\
\text { gastrostomia percutânea endoscópica foi a forma } \\
\text { de paliação nutricional, sem expectativa de } \\
\text { retomar a ingestão oral. }\end{array}$ & $\begin{array}{l}\text { Uso de terapia nutricional enteral ou } \\
\text { parenteral. }\end{array}$ & $\begin{array}{l}\text { Em pacientes com câncer avançado do esôfago, em que só a terapêutica paliativa é } \\
\text { possível, o suporte nutricional é facilmente obtido com gastrostomia percutânea } \\
\text { endoscópica, permitindo aos pacientes permanecerem em suas casas por um longo } \\
\text { período. A nutrição por gastrostomia percutânea endoscópica deveria ser considerada, } \\
\text { por rotina, como a opção definitiva para paliação nutricional em pacientes com câncer } \\
\text { do esôfago proximal em que a colocação de prótese não é possível. }\end{array}$ \\
\hline $\begin{array}{l}\text { Kapoor et } \\
\text { al, } 2017\end{array}$ & $\begin{array}{l}\text { Determinar se a intervenção nutricional } \\
\text { (suplementação com IAtta }+ \text { aconselhamento } \\
\text { dietético }+ \text { atividade física) melhora os } \\
\text { indicadores antropométricos e a qualidade de vida } \\
\text { em pacientes com câncer submetidos a cuidados } \\
\text { paliativos. }\end{array}$ & $\begin{array}{l}\text { Contribuições da suplementação } \\
\text { nutricional; } \\
\text { Estado Nutricional. }\end{array}$ & $\begin{array}{l}\text { Os pacientes do grupo controle (aconselhamento nutricional }+ \text { atividade física) } \\
\text { apresentaram redução significativa do peso corporal, circunferência do braço - parte } \\
\text { superior do braço gordura corporal ao final da intervenção. Foi observada uma } \\
\text { tendência de ganho de peso corporal no grupo de intervenção e aumento significativo } \\
\text { de gordura corporal. Além disso, os pacientes relataram uma melhora significativa na } \\
\text { fadiga e escores de apetite nos domínios de qualidade de vida no final da intervenção. } \\
\text { De forma que a incorporação da suplementação nutricional na terapia de cuidados } \\
\text { paliativos pode melhorar a qualidade de vida e estabilizar o peso corporal em pacientes } \\
\text { com caquexia por câncer. }\end{array}$ \\
\hline $\begin{array}{l}\text { Orrevall et } \\
\text { al, } 2013\end{array}$ & $\begin{array}{l}\text { Investigar a prevalência e uso de TNE, TNP e } \\
\text { glicose intravenosa em pacientes diagnosticados } \\
\text { com câncer que foram inscritos em serviços } \\
\text { especializados de cuidados paliativos para } \\
\text { pacientes internados e domiciliares em toda a } \\
\text { Suécia. Além de investigar as indicações e } \\
\text { benefícios da nutrição artificial e glicose } \\
\text { intravenosa em relação à sobrevida prevista. }\end{array}$ & $\begin{array}{l}\text { Uso de terapia nutricional enteral ou } \\
\text { parenteral. }\end{array}$ & $\begin{array}{l}\text { O tratamento com TNP foi mais comum do que a TNE, porém foram observadas } \\
\text { diferenças significativas no uso de TNP dentro do país. Essas diferenças, tanto entre } \\
\text { regiões urbanas e rurais, bem como em uso de PN entre diferentes configurações de } \\
\text { cuidados paliativos urbanos, exigem uma investigação mais aprofundada. Em relação } \\
\text { aos benefícios do suporte nutricional como nenhum critério foi fornecido para essa } \\
\text { avaliação, o entendimento foi limitado, indicando que os resultados devem ser } \\
\text { interpretados com cautela. }\end{array}$ \\
\hline
\end{tabular}




\begin{tabular}{|c|c|c|c|}
\hline $\begin{array}{l}\text { Pelzer et al, } \\
2010\end{array}$ & $\begin{array}{l}\text { No presente estudo, investigou-se o impacto de } \\
\text { nutrição parenteral adicionais sobre o estado } \\
\text { nutricional em pacientes com câncer avançado de } \\
\text { pâncreas, usando parâmetros da BIA como o } \\
\text { ângulo de fase, o índice de ECM / BCM e IMC. }\end{array}$ & $\begin{array}{l}\text { Uso de terapia nutricional enteral ou } \\
\text { parenteral. }\end{array}$ & $\begin{array}{l}\text { A maioria dos pacientes que receberam nutrição parenteral (NP) mostrou um estado } \\
\text { nutricional melhorado, o que foi verificado por alterações nos parâmetros-alvo. O que } \\
\text { demostra que há impacto positivo da NP no estado nutricional de pacientes com com } \\
\text { câncer avançado de pâncreas. }\end{array}$ \\
\hline $\begin{array}{l}\text { Queiroz et } \\
\text { al, } 2018\end{array}$ & $\begin{array}{l}\text { Avaliar a prevalência de sarcopenia e sua } \\
\text { associação com estado nutricional e qualidade de } \\
\text { vida em pacientes com câncer avançado em } \\
\text { cuidados paliativos. }\end{array}$ & Estado Nutricional. & $\begin{array}{l}\text { Constatou-se dinapenia em } 21,9 \% \text {, a atrofia muscular em } 17,1 \% \text { e a sarcopenia, } \\
\text { caracterizada pela associação de ambas as condições, em } 32,4 \% \text { da amostra estudada. } \\
\text { Todas as variáveis antropométricas foram significativamente menores nos indivíduos } \\
\text { sarcopênicos, além disso, indivíduos sarcopênicos possuíam funcionalidade } \\
\text { prejudicada. Portanto é importante a uniformização dos critérios diagnósticos e a } \\
\text { elaboração de protocolos de assistência nutricional específicos para pacientes } \\
\text { oncológicos em cuidado paliativo, de modo a resultar em melhora da qualidade de } \\
\text { vida. }\end{array}$ \\
\hline $\begin{array}{l}\text { Silva et al, } \\
2010\end{array}$ & $\begin{array}{l}\text { Analisar como a intervenção nutricional e o } \\
\text { controle dos sintomas interferiram na qualidade de } \\
\text { vida dos pacientes oncológicos atendidos pelo } \\
\text { Serviço de Cuidados Paliativos (CP) e Tratamento } \\
\text { da Dor do Hospital Erasto Gaertner. }\end{array}$ & Acompanhamento nutricional. & $\begin{array}{l}\text { Concluiu-se que uma intervenção médica e nutricional de forma integrada contribuiu } \\
\text { para o controle dos sintomas, e isso possibilita a discussão quanto à conduta } \\
\text { individualizada, promovendo o controle adequado da dor, aumento da ingestão } \\
\text { alimentar e auxiliando o paciente a viver com melhor qualidade de vida. }\end{array}$ \\
\hline $\begin{array}{c}\text { Vetori, } \\
\text { Santos e } \\
\text { Peria, } 2018\end{array}$ & $\begin{array}{l}\text { Caracterizar o diagnóstico e o cuidado nutricional } \\
\text { em pacientes em cuidados paliativos } \\
\text { hospitalizados em um serviço de saúde pública. }\end{array}$ & Acompanhamento nutricional. & $\begin{array}{l}\text { Neste estudo, a maioria da população encontrava-se desnutrida, com importantes } \\
\text { déficits nutricionais. Constatou-se que a oferta de energia e macronutrientes foi uma } \\
\text { prioridade na terapia nutricional, excedendo as necessidades nutricionais estimadas e } \\
\text { que o jejum não se correlacionou com a morte ou déficit nutricional no estágio final } \\
\text { da vida. }\end{array}$ \\
\hline
\end{tabular}

